\title{
Diagnóstico del sistema de instrucción virtual del área de ciencias de la salud de la Escuela de Física de la UNAH durante la pandemía Covid-19
}

\author{
Diagnosis of the virtual instruction system of the health sciences area of the \\ School of Physics at UNAH during the Covid-19 pandemic
}

F. BARRALAGA ${ }^{1}$

Recibido: 15 de octubre de 2020 / Aceptado: 18 de diciembre de 2020

\begin{abstract}
RESUMEN
La adaptibilidad del sistema de instrucción educativa se encuentra en proceso de evolución en todo el mundo para evitar el estancamiento educativo. La pandemia de Covid-19 ha obligado a cambiar los procesos de enseñanza y rápidamente se están produciendo innovaciones en la forma de instrucción docente, que garanticen la calidad de la instrucción y el alcance de los objetivos planteados en las planificaciones originales. Un sistema educativo tiene múltiples facetas y todas deben ser consideradas en un tiempo corto, para plantear soluciones adecuadas e inmediatas. La Escuela de Física de la Universidad Nacional Autónoma de Honduras, dió seguimiento a la adaptabilidad de los estudiantes del área de la salud atendidos por esta unidad durante el segundo período del 2020, mediante una consulta aplicada a una muestra de esta población estudiantil, que auscultaba los aspectos más relevantes de su proceso de instrucción emergente. Una consulta similar se había realizado a los estudiantes del área fisico-matemáticas, que fueron atendidos durante el primer período del mismo año. Los resultados de esta última consulta son expuestos y se establecen algunas comparaciones con los obtenidos en la primera.
\end{abstract}

\begin{abstract}
The adaptability of the educational instruction system is in process of evolution throughout the world, to avoid educational stagnation. The Covid-19 pandemic has forced changes in teaching processes and innovations are rapidly taking place in the form of teacher instruction, which guarantee the quality of instruction and the achievement of the objectives set out in the original plans. An education system has multiple facets and all of them must be considered in a short time, to propose adequate and immediate solutions. The School of Physics of the National Autonomous University of Honduras, followed up on the adaptability of the students in the health area attended by this unit, during the second period of 2020, through a consultation applied to a sample of this student population, which monitored the most relevant aspects of the emergent instructional process. A similar consultation had been made to the students in the physical-mathematical area, that were attended during the first period of the same year. The results of this last consultation are exposed and some
\end{abstract}

${ }^{1}$ Escuela de Física, Facultad de Ciencias,

Universidad Nacional Autónoma de Honduras email: francisco.barralaga@unah.edu.hn 
comparisons are established with those obtained in the first consultation.

\section{PALABRAS CLAVES}

Instrucción virtual, adaptabilidad ante Covid-19, resiliencia educativa

KEYWORDS

Virtual instruction, adaptability against Covid-19, educative resilience

\section{I | INTRODUCCIÓN}

La consulta realizada por la UNESCO a diecisiete países de América Latina (UNESCO, 2020), incluido Honduras, para conocer la condición de afrontamiento de sus sistemas educativos ante la crisis del Covid-19, muestra que la generalidad de éstos ha tomado soluciones temporales, y a corto plazo trabajan en definir y mejorar sus metodologías de enseñanza virtual y en la búsqueda de retornos seguros a la modalidad de educación presencial.

La Escuela de Física de la Universidad Nacional Autónoma de Honduras, UNAH, realizó el seguimiento de la condición estudiantil en relación al sistema de instrucción resultante de la pandemia, primero, durante el primer período del 2020, consultando a una muestra de la población del área físico-matemática (ciencias e ingenierías) y posteriormente, durante el segundo período del mismo año, auscultando a una muestra de la población de estudiantes del área de la salud (ciencias médicas y biológicas).

Las consultas pretenden obtener información sobre la percepción estudiantil de la metodología emergente implementada, el acceso a la comunicación por internet, y el uso, por parte de los docentes, de herramientas tecnológicas en la enseñanza virtual.

La muestra consultada incluyó estudiantes que cursaban las asignaturas de Física General (FS104), Física Médica (FS111), Biofísica (FS210), evaluándose los aspectos más relevantes del proceso de instrucción. Se incluyen en este artículo los resultados obtenidos más importantes, además de la comparación de estos con los correspondientes a la consulta realizada a los estudiantes del área físicomatemática durante el período académico previo. Se espera que estos resultados puedan ser útiles a los tomadores de decisión del ámbito educativo, para adoptar las mejores opciones de solución para el abordaje de esta crisis

\section{I DESARROLLO DEL TEMA}

Esta investigación cuali-cuantitative $2^{1}$ fue realizada a una población estudiantil de 1200 estudiantes que cursaban las asignaturas de Física del área de Salud. La Figura 1 detalla las asignaturas y la distribución porcentual de la muestra.

La consulta fue diseñada para obtener respuestas anónimas y consta de treinta y dos (32) preguntas que evaluán la percepción de los estudiantes en relación a los siguientes aspectos relevantes de la instrucción adoptada por la Escuela de Física de la UNAH, resultante de la crisis de la pandemia Covid-19:

\footnotetext{
${ }^{1}$ incluía tanto preguntas abiertas como de valoración numérica
} 


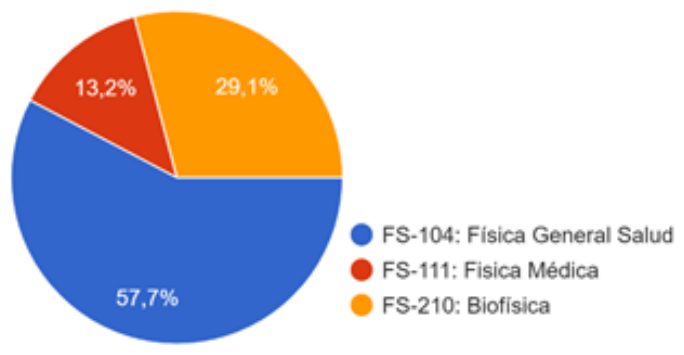

Figura 1: Distribución porcentual de la muestra.

- Equipo de cómputo y conexión a internet,

- Competencias requeridas,

- Técnología de comunicación,

- Desempeño de plataforma tecnológica,

- Posibles herramientas de instrucción.

El cálculo de la muestra se obtuvo a partir de la fórmula para población finita siguiente (Pedro López-Roldán, 2015):

$$
\mathrm{n}=\frac{\sigma^{2} z^{2} N}{e^{2}(N-1)+\sigma^{2} z}
$$

Con:

$$
\begin{aligned}
e & =0.09, \\
\sigma & =0.5, \\
N & =1200, \\
z & =1.96
\end{aligned}
$$

Resultó una muestra de 108 estudiantes; contestaron 184 estudiantes de la población especificada. A continuación se incluyen los resultados más relevantes, y su comparación con la consulta practicada a la población estudiantil del área físico-matemática.

\section{III | RESULTADOS OBTENIDOS}

Casi el $58 \%$ de la muestra correspondió a estudiantes de Física General para la Salud (FS104), que tradicionalmente es la asignatura más numerosa de ésta área académica. Esta asignatura es cursada por estudiantes de las facultades de Odontología, Farmacia, y de las Escuelas de Biología y Microbiología pertenecientes a la Facultad de Ciencias; el $42.3 \%$ fueron estudiantes de la Facultad de Ciencias Médicas, $13.2 \%$ de Física Médica (FS111) y 29.1\% de Biofísica (FS2010). Los resultados de la consulta a esta muestra se detallan a continuación en las diferentes secciones de la consulta. 


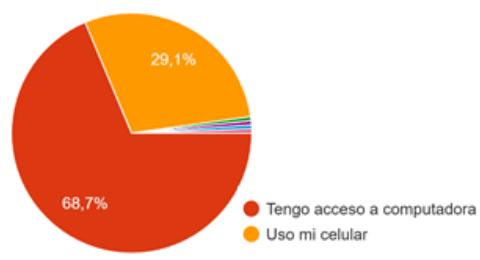

Figura 2: Disponibilidad de equipo de computo.

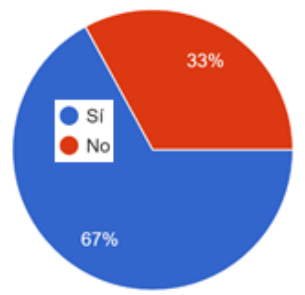

Figura 5: Disposición de competencias para la instrucción virtual.

Figura 6: Nivel de aprendizaje autónomo.
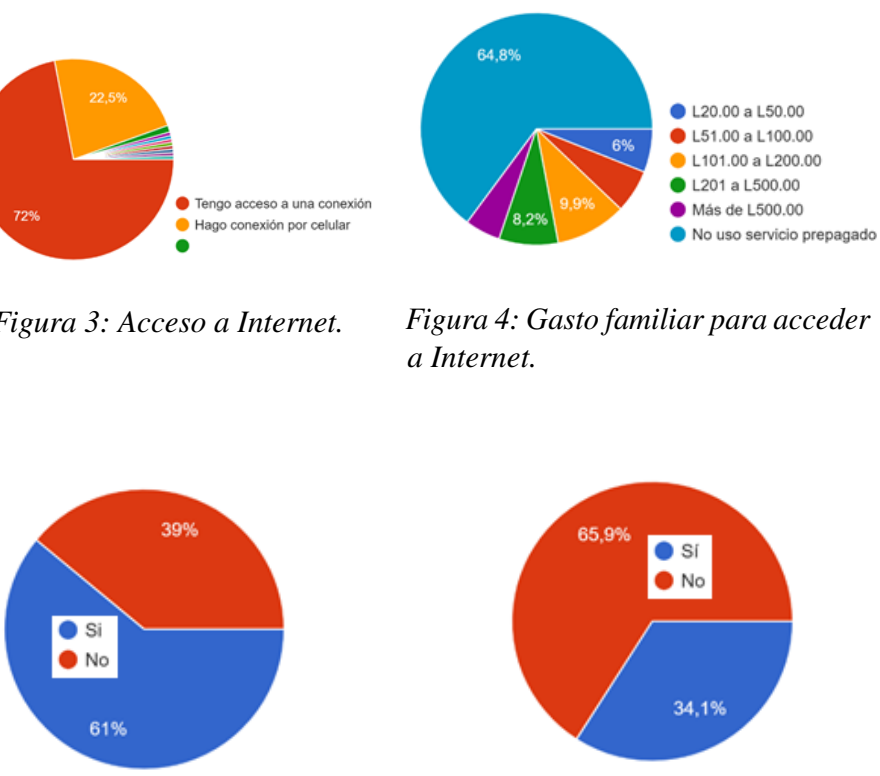

Figura 3: Acceso a Internet.

Figura 4: Gasto familiar para acceder a Internet.

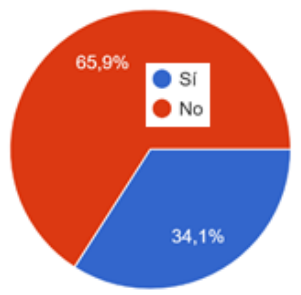

Figura 7: Manejo de software de instrucción.

\section{1 | Equipo y Conexión de Internet}

El $68.7 \%$ de la muestra tiene acceso a una computadora mientras que el $29.1 \%$ hace uso de su celular como equipo de acceso. Esto está evidenciado en la Figura2

Por otra parte, la información de la Figura 3 indica que el $72 \%$ de la muestra tiene conexión de internet via Wifi, un $22.5 \%$ accede a internet a través de su celular debiendo hacer compras de paquetes de datos de pre-pago para comunicarse por internet. Obsérvese (Figura 4) que un $33.2 \%$ invierte entre L20.00 a L500.00 semanales adquiriendo estos paquetes de pre-pago para poder realizar las actividades exigidas por el sistema de instrucción.

\section{2 | Competencias requeridas}

Los estudiantes de esta área académica tienden a tener mayores dificultades con las asignaturas relacionadas con matemáticas; su percepción en cuanto a sus competencias necesarias para llevar a cabo una instrucción virtual, para la que se requiere una independencia académica mayor y un buen nivel de autodidaxia, se evidencia en la Figura 5 en la que el $67 \%$ afirma disponer de las competencias suficientes para afrontar este tipo de instrucción, y el $61 \%$ afirma tener un nivel adecuado de autodidaxia (Figura 6). Adicionalmente el 65.9\% como indica la Figura 7, manifiesta no tener dificultades para el manejo del software involucrado en la instrucción virtual. 


\begin{tabular}{lll}
\hline Herramientas de Instrucción & $\mathbf{f}$ & $\%$ \\
\hline Vídeos & 52 & $29.71 \%$ \\
Tareas & 37 & $21.14 \%$ \\
Lecturas & 23 & $13.14 \%$ \\
Control de Vídeos & 20 & $11.43 \%$ \\
Foros & 17 & $9.71 \%$ \\
Simulaciones & 15 & $8.57 \%$ \\
Control de Lecturas & 11 & $6.29 \%$ \\
\hline TOTALES & 175 & $100.00 \%$ \\
\hline
\end{tabular}

Tabla 1: Evaluación de herramientas de IV.

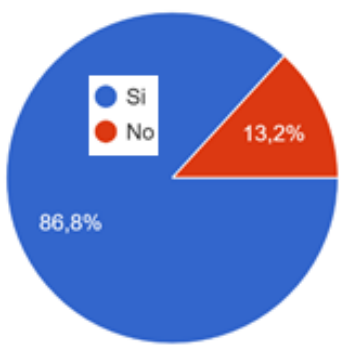

Figura 8: Uso de comunicación sincrónica.

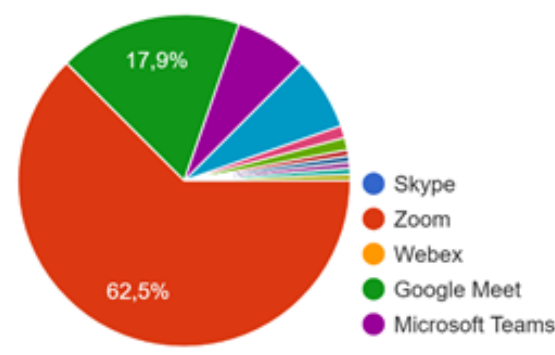

Figura 9: Aplicaciones usadas en comunicación síncrona.

\section{3 | Tecnológia de Comunicación}

Para este segundo período, ya el $86.8 \%$ de los docentes utilizaban tecnología de comunicación sincrónica para impartir sus clases, como afirman los estudiantes según la gráfica de la Figura 8 Según la Figura 9. el $62.5 \%$ utilizan Zoom, la más utilizada, seguida de Google Meet con 17.9\% y Teams con 14.2\%; el resto utilizan otras herramientas. Otras aplicaciones que los docentes usan para complementar la comunicación con sus estudiantes se muestran en la Figura 10

\section{4 | Plataforma Tecnológica}

La UNAH dispone de una plataforma tecnológica basada en Moodle y da acceso a comunicación síncrona a través de Zoom y de Team. El despliegue gráfico de la Figura 11, señala que más del 96.2\% de los docentes usan esta plataforma para agregar contenido y actividades asincrónas en el desarrollo de su clase.

Las herramientas de instrucción provistas por esta plataforma que los docentes de esta área utilizan, se señalan en la Figura 12 , éstas son: tareas, pruebas en línea, seguidas de vídeos, lectura y simulaciones.

La percepción de la muestra en relación a grado de aprendizaje de estas herramientas, se manifiesta en 


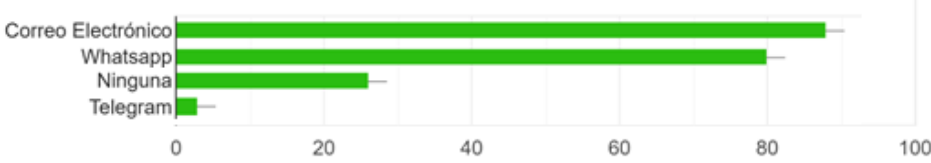

Figura 10: Herramientas complementarias de comunicación.

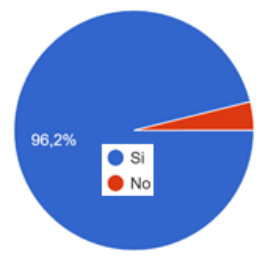

Figura 11: Uso de la plataforma de la UNAH.

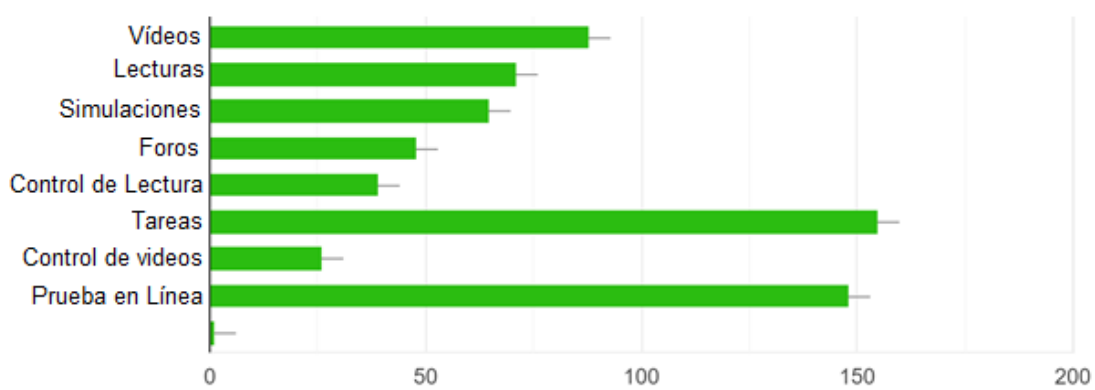

Figura 12: Opciones de Moodle utilizadas en la instrucción virtual.

la Figura 13 que indica la validación obtenida para cada una; puede verificarse que los vídeos fueron considerados los más efectivos, seguidos de tareas, lecturas, controles de visualización de vídeos, foros, simulaciones y, considerados los de menor efectividad, los controles de lectura. Un detalle de esta evaluación se incluye en la Tabla 3

El $84.1 \%$ de la muestra opina que el docente use tanto la plataforma de la UNAH para incluir actividades asíncronas, como alguna tecnología de videoconferencia para la comunicación síncrona para sus clases. Esto queda evidenciado en la Figura 14.

\section{5 | Posibles Herramientas de Instrucción}

Se presentaron a la muestra cuatro (4) herramientas de instrucción, para evaluar su posible uso en los siguientes períodos académicos. Estas fueron:

- Pizarra virtual: software que permite utilizar la pantalla de la computadora como una pizarra y desplegar gráficos, ecuaciones y otros contenidos multimedia.

- Vídeo con control de atención: vídeo sobre un tópico, que plantea preguntas sobre el contenido expuesto, para evaluar el nivel de atención.

- Visualización de proceso escrito: una cámara (usualmente de un celular), permite a los estudiantes visualizar un procedimiento de solución resuelto por el docente en papel.

- Simulación explicada: uso de simulaciones en computadora sobre algún fenómeno físico, utilizado como complemento para detallar el desarrollo de un tema. 


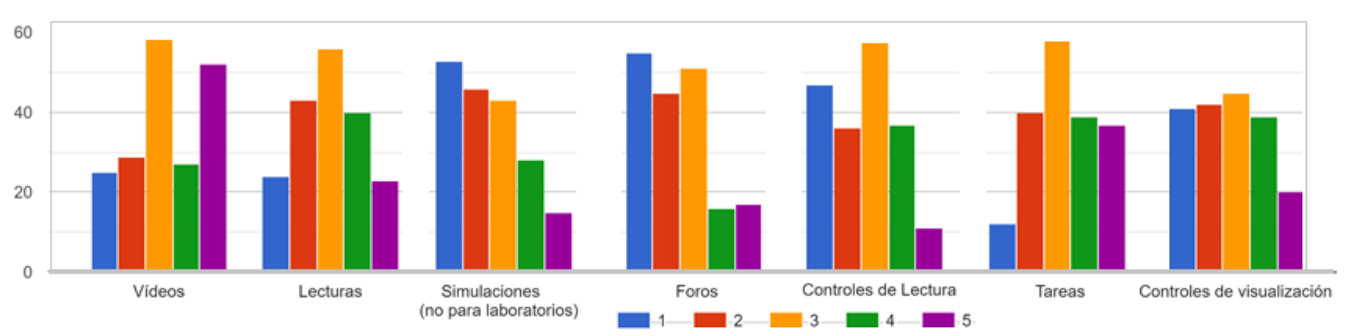

Figura 13: Potencial de aprendizaje de herramientas de instrucción.

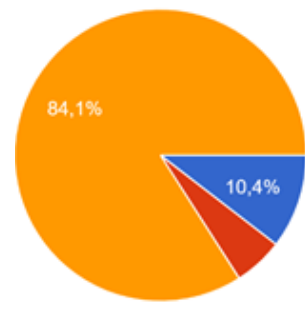

Que el docente SOLO haga uso de la Plataforma Virtual como herramienta para realizar su instrucción

Q Que el docente SOLO haga uso de tecnología de video-conferencia e imparta su instrucción diariamente en el horario original de la clase

Que el docente use AMBAS opciones como herramientas de instrucción

Figura 14: Preferencias sobre la modalidad de instrucción.

La Figura 15 muestra el resultado de la validación de estas opciones por parte de los estudiantes consultados. Claramente ellos consideran con el mayor grado de efectividad para el aprendizaje a la pizarra virtual, seguida de la simulación explicada, y la visualización de proceso escrito la tercera; el video con control de atención incrustado resultó ser el de menor nivel de aprendizaje.

\section{6 | Evaluación de Plataforma Tecnológica}

La evaluación obtenida por la plataforma tecnológica de la UNAH por parte de la muestra se indica en la Figura 16 Un análisis con algoritmos de minería de datos de la librería Machine Learning NLTK de Python5 (Bengfort, 2016, Brownlee, 2017; Gonzáles, 2020), se realizó a las respuestas abiertas que justifican esta calificación, y el mismo mostró que las palabras que más se repiten en estas respuestas son las indicadas, junto con su porcentaje de abundancia en la Tabla 2

\section{7| Comparación con Área Físico-Matemática}

La Tabla 3 detalla la comparación entre los aspectos más relevantes de los sectores estudiantiles de las áreas Físico-Matemático (FM) y Ciencias de la Salud (CS) de la Escuela de Física.

\section{I CONCLUSIONES}

1. Similarmente a los estudiantes del área Físico-Matemáticas, cerca del $70 \%$ de la muestra tiene acceso a computadora y casi un $30 \%$ usa su celular para realizar sus actividades de instrucción.

2. Para el segundo período, el $72 \%$ de los estudiantes tienen acceso a Internet vía WiFi. Esto es un incremento del $17 \%$ respecto de la muestra de Físico Matemáticas que fue consultada a finales del 


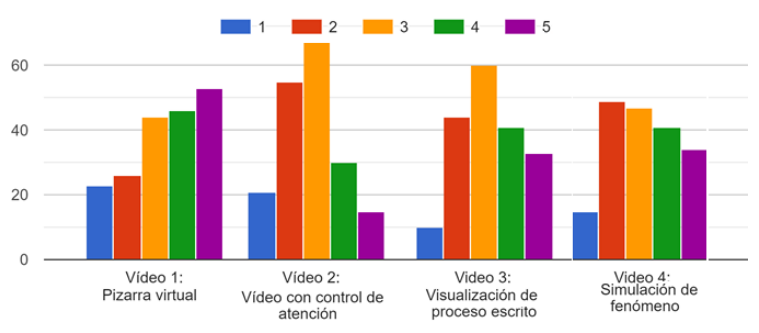

Figura 15: Futuras herramientas de instrucción.

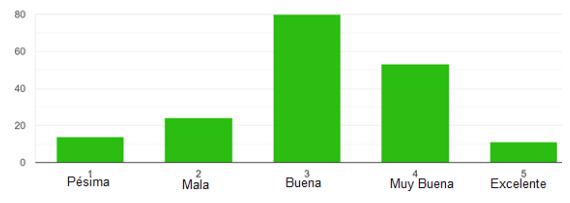

Figura 16: Desempeño de plataforma de la UNAH.

\begin{tabular}{clcc}
\hline No.1 & Palabra & $\mathrm{f}$ & $\%$ \\
\hline 1 & Cae & 18 & \\
2 & Problemas & 13 & $74.58 \%$ \\
3 & Falla & 13 & \\
\hline 4 & Bien & 8 & $25.42 \%$ \\
5 & Buena & 7 & \\
\hline \multicolumn{2}{c}{ TOTALES } & 59 & $100.00 \%$ \\
\hline
\end{tabular}

Tabla 2: Evaluación de plataforma-UNAH

primer período académico del 2020.

3. Un $35.2 \%$ de los estudiantes de la muestra invierten entre L20.00 a L500.00 semanalmente. En comparación, la muestra de Físico-Matemática en la que el $43.9 \%$ hacia esta inversión.

4. El $65.2 \%$ de la muestra, dice manejar adecuadamente las herramientas de instrucción virtual. En comparación, el 84.2\% del área Físico-Matemáticas tiene estas competencias.

5. Para este segundo período, el $86.8 \%$ de los docentes dan sus clases haciendo uso de alguna aplicación de comunicación síncrona. Un incremento de $23.6 \%$ sobre el primer período.

6. Más del $96 \%$ de los docentes usan la plataforma de la UNAH para la comunicación asíncrona con sus estudiantes.

7. Las herramientas de la Plataforma Virtual que los docentes usan, son, en orden de mayor a menor frecuencia: Tareas, Pruebas en Línea, vídeos, lecturas, simulaciones (no para laboratorio), Foros y controles de lectura.

8. En cuanto a la efectividad didáctica de estas herramientas, la muestra considera como más efectiva a los vídeos, seguidos de tareas, lecturas, controles de visualización de vídeos, simulaciones (usadas en laboratorios) y foros; opinan que los controles de lectura son los menos efectivos.

9. La herramienta de instrucción virtual que podría usarse y que la muestra consideró con mayor potencial de aprendizaje es la Pizarra Virtual, seguida de Simulaciones Explicadas, y la Visualización de Proceso Escrito. 


\begin{tabular}{lcc}
\hline \multicolumn{1}{c}{ Consulta } & FM & CS \\
\hline Acceso a computadora & $70.80 \%$ & $68.70 \%$ \\
Acceso a WiFi & $54.90 \%$ & $72.00 \%$ \\
Usa Pre-Pago para IV & $43.90 \%$ & $35.20 \%$ \\
Maneja herramientas de IV & $84.20 \%$ & $65.90 \%$ \\
Es autodidacta & $53.2 \%$ & $61 \%$ \\
Tiene competencias para IV & $65.50 \%$ & $67.00 \%$ \\
Dominio de Inglés & $50.90 \%$ & $53.30 \%$ \\
Docentes usan CS en IV & $63.20 \%$ & $86.80 \%$ \\
Aplicación de CS más usada & Zoom & Zoom \\
Docentes usan otras ACA & $85.20 \%$ & $85.50 \%$ \\
Docentes usan LMS-UNAH & $97.10 \%$ & $96.20 \%$ \\
Prefieren tanto CS y CA & $86.40 \%$ & $84.10 \%$ \\
Nueva herramienta de IV & PV & PV \\
\hline
\end{tabular}

Tabla 3: Comparación FM y CS. Abreviatura usada, LMS: Learning Management System, ACA: Aplicación de comunicación asíncrona, CS: Comunicación síncrona, CA: Comunicación asíncrona, IV: Instrucción virtual, FM: Físico-matemática, CS: Ciencias de la salud.

10. El desempeño de la plataforma de la UNAH fue considerada regular,similarmente a la evaluación que hicieron los estudiantes a finales del primer período. Esto implica, que las condiciones de esta plataforma, para finales del segundo período no habían cambiado a pesar de las múltiples notificaciones por parte de las autoridades académicas a la unidad a cargo.

11. Tanto los estudiantes del área de Ciencias de la Salud como los del área Físico-Matemática, opinan que el uso de las pizarras virtuales, mejoraría el nivel de aprendizaje del sistema de instrucción. La segunda herramienta que consideraron adecuada para lograr esta mejoría son las simulaciones, incorporadas en las comunicaciones síncronas para describir los fenómenos físicos tratados.

\section{REFERENCIAS}

Bengfort, B. (2016). Applied text analysis with python. O'Reilly.

Brownlee, J. (2017). Deep learning for natural language processing. Machine Learning Mastery.

Gonzáles, C. G. (2020). Clasificador de texto mediante técnicas de aprendizaje automático. Valencia: Universitat Politècnica de València.

Pedro López-Roldán, S. F. (2015). Metodología de la investigación social cuantitativa. Barcelona: UAB. Universitat Autònoma de Barcelona.

UNESCO. (2020). Evaluación y continuidad educativa en momentos difíciles. 